\title{
Advances in gout: some answers, more questions
}

\author{
Jasvinder A Singh*1-4 \\ See related research by Becker et al., http://arthritis-research.com/content/12/2/R63
}

\begin{abstract}
In a previous issue of the journal, Becker and colleagues present efficacy and safety data from a large study comparing febuxostat to allopurinol. The study showed non-inferiority of febuxostat $40 \mathrm{mg} /$ day in lowering serum urate compared to allopurinol 200 to $300 \mathrm{mg} /$ day. More importantly, the study showed a similar frequency of important cardiovascular adverse events (cardiovascular death, nonfatal myocardial infarction and nonfatal stroke) for febuxostat $40 \mathrm{mg} /$ day (0\%), febuxostat $80 \mathrm{mg} /$ day (0.4\%) and allopurinol groups (0.4\%). Other cardiac adverse event rates (unstable angina, coronary revascularization, cerebral revascularization, transient ischemic attack, venous and peripheral arterial vascular thrombotic event, congestive heart failure, and arrhythmia) were also similar for febuxostat $40 \mathrm{mg} /$ day (1.3\%), febuxostat $80 \mathrm{mg} /$ day (1.2\%) and allopurinol groups (0.9\%). A meta-analysis of safety data from published studies is presented.
\end{abstract}

For many decades, urate-lowering therapy consisted of allopurinol, a xanthine-oxidase inhibitor and uricosurics, such as probenecid, sulfinpyrazone and benzbromarone. Febuxostat, another xanthine inhibitor, was approved in 2009 in the US, Canada, Japan and the European Union for the treatment of hyperuricemia in gout patients. Gout is associated with high medical comorbidity and deficits in health-related quality of life. In a previous issue of Arthritis Research and Therapy, Becker and colleagues [1] describe a non-inferiority randomized controlled trial (RCT) of febuxostat compared to allopurinol in 2,269 patients with gout. Several findings of this important study merit discussion.

The cornerstone of gout treatment is achievement of a target serum urate $<6 \mathrm{mg} / \mathrm{dl}$. This therapeutic goal is

\footnotetext{
*Correspondence: Jasvinder.md@gmail.com

'Medicine Service, Birmingham VA Medical Center and Department of Medicine,

University of Alabama, Birmingham, AL, USA
}

Full list of author information is available at the end of the article based on the solubility of urate at $37^{\circ} \mathrm{C}(6.8 \mathrm{mg} / \mathrm{dl})$, levels below which have been associated with lower risk of gout flares and tophi [2-4]. A target serum urate was achieved in significantly more patients in the febuxostat $80 \mathrm{mg}$ group (67\%) compared to the $40 \mathrm{mg}$ group (45\%) and the allopurinol group (42\%; $300 \mathrm{mg}$ daily or $200 \mathrm{mg}$ daily in moderate renal impairment); the $40 \mathrm{mg}$ febuxostat dose was not inferior to allopurinol [5]. First, the superiority of febuxostat $80 \mathrm{mg} /$ day to allopurinol $300 \mathrm{mg} /$ day in achieving serum urate $<6 \mathrm{mg} / \mathrm{dl}$ in this study confirms similar findings from previous RCTs of febuxostat using this or higher doses of febuxostat (120 and $240 \mathrm{mg}$ daily) [5-7]. This study provides evidence for non-inferiority of a febuxostat $40 \mathrm{mg}$ dose compared to allopurinol in achieving a target serum urate $<6 \mathrm{mg} / \mathrm{dl}$. One important fact to bear in mind, however, is that the allopurinol dose should be optimally titrated between 100 and $800 \mathrm{mg} /$ day in clinical settings (or even higher doses in some cases according to experts) to achieve a target serum urate $<6 \mathrm{mg} / \mathrm{dl}$, and all febuxostat studies to date have used 200 or $300 \mathrm{mg}$ allopurinol as the comparator. Therefore, these studies do not answer an important question: is febuxostat superior to a titrated dose of allopurinol in the treatment of hyperuricemia in patients with gout? We know now that allopurinol doses of $\leq 300 \mathrm{mg} /$ day fail to adequately treat hyperuricemia in $50 \%$ of gout patients [8]. It should be noted that, in practice, many physicians do not adequately titrate allopurinol and fail to follow serum urate to achieve the target level [9-10]. Thus, there is limited 'real world logic' in such a study design strategy.

Second, this study provides safety data as one of its important secondary outcomes. This finding has high relevance since cardiovascular adverse outcomes were an early safety signal in the first large febuxostat RCT, with four deaths in the two febuxostat groups and no deaths in the allopurinol group [5]. In the current study, febuxostat was well-tolerated and not associated with significantly more cardiovascular adverse events compared to allopurinol [1]. Cardiovascular adverse events as defined by the Adjudicated Antiplatelet Trialists Collaboration (APTC; cardiovascular death, nonfatal myocardial infarction, and nonfatal stroke) occurred in $0 \%$ of patients in the febuxostat $40 \mathrm{mg}$ group, $0.4 \%$ in the febuxostat $80 \mathrm{mg}$ group and $0.4 \%$ in the allopurinol group, without a 
Table 1. Serious adverse events, cardiovascular adverse events and mortality in randomized controlled trials of febuxostat

\begin{tabular}{|c|c|c|c|c|c|c|}
\hline Study & Duration & $\begin{array}{l}\text { Febuxostat } \\
40 \mathrm{mg} / \text { day }\end{array}$ & $\begin{array}{l}\text { Febuxostat } \\
80 \mathrm{mg} / \text { day }\end{array}$ & $\begin{array}{l}\text { Febuxostat } \\
120 \mathrm{mg} / \text { day }\end{array}$ & $\begin{array}{c}\text { Allopurinol } \\
200 / 300 \mathrm{mg} / \text { day }\end{array}$ & Placebo \\
\hline \multicolumn{7}{|c|}{ Cardiovascular adverse events } \\
\hline Schumacher et al. [7] & 1 month & - & $5 / 267(2 \%)^{a}$ & $5 / 269(2 \%)$ & $1 / 268(0.4 \%)$ & $1 / 134(0.7 \%)$ \\
\hline Current study [1] & 6 months & $\begin{array}{c}\text { APTC: } 0 / 757(0 \%) \\
\text { Non-APTC: } 10 / 757(1.3 \%)\end{array}$ & $\begin{array}{c}\text { APTC: } 3 / 756(0.4 \%) \\
\text { Non-APTC: } 9 / 756(1.2 \%)\end{array}$ & - & $\begin{array}{c}\text { APTC: } 3 / 756(0.4 \%) \\
\text { Non-APTC: } 7 / 756(0.9 \%)\end{array}$ & - \\
\hline
\end{tabular}

Death

\begin{tabular}{|c|c|c|c|c|c|c|}
\hline Schumacher et al. [7] & 1 month & - & $0 / 267(0 \%)^{a}$ & 0/269 (0\%) & 0/268 (0\%) & 0/134 (0\%) \\
\hline Becker et al. [6] & 1 month & 0/37 (0\%) & $0 / 40(0 \%)$ & 0/38 (0\%) & - & $0 / 38(0 \%)$ \\
\hline Current study [1] & 6 months & 1/757 (0.1\%) & 1/756 (0.1\%) & - & 3/756 (0.4\%) & - \\
\hline Becker et al. [5] & 1 year & - & $2 / 256(1 \%)$ & $2 / 251(1 \%)$ & 0/253 (0\%) & - \\
\hline
\end{tabular}

Serious adverse events as defined in each study ${ }^{b}$

\begin{tabular}{|c|c|c|c|c|c|c|}
\hline Schumacher et al. [7] & 1 month & - & $11 / 267(4 \%)^{\mathrm{a}}$ & $9 / 269(3 \%)$ & $7 / 268(3 \%)$ & $2 / 134(1 \%)$ \\
\hline Becker et al. [6] & 1 month & 0/37 (0\%) & $1 / 40(2.5 \%)$ & $2 / 38(5.5 \%)$ & - & 0/38 (0\%) \\
\hline Current study [1] & 6 months & $19 / 757(2.5 \%)$ & 28/756 (3.7\%) & - & $31 / 756(4.1 \%)$ & - \\
\hline Becker et al. [5] & 1 year & - & $11 / 256(4.3 \%)$ & 21/251 (8.4\%) & 19/253 (7.5\%) & - \\
\hline
\end{tabular}

ane out of 134 (1\%) had cardiovascular adverse events, 5 out of 134 (4\%) had serious adverse events and none of the patients died in the 240 mg febuxostat group in the study by Schumacher and colleagues [7]. Adjudicated Antiplatelet Trialists Collaboration (APTC) events were defined as one or more of the following: cardiovascular death, nonfatal myocardial infarction, and nonfatal stroke. Non-APTC cardiovascular events included unstable angina, coronary revascularization, cerebral revascularization, transient ischemic attack, venous and peripheral arterial vascular thrombotic event, congestive heart failure, and arrhythmia. ${ }^{\text {SSerious }}$ adverse events were defined as follows in each study: no definitions were provided in the Schumacher and colleagues [7], Becker and colleagues [6] or the current study [1]; Becker and colleagues [5] defined a serious adverse event as '.... an event that was life-threatening or that resulted in death, hospitalization or prolongation of hospitalization, persistent disability or incapacity, or a congenital anomaly or birth defect.'

statistically significant difference; non-APTC events (unstable angina, coronary revascularization, cerebral revascularization, transient ischemic attack, venous and peripheral arterial vascular thrombotic event, congestive heart failure, and arrhythmia) occurred in 1.3\%, 1.2\% and $0.9 \%$, respectively. A review of RCTs of febuxostat showed that cardiovascular adverse events were reported in 1.3 to $2 \%$ of febuxostat-treated patients versus 0.4 to $0.9 \%$ of allopurinol-treated patients, and death occurred in 0 to $1 \%$ of febuxostat-treated patients versus 0 to $0.4 \%$ of allopurinol-treated patients (Table 1). These differences were not statistically significantly different in each study.

A meta-analysis of data provided in Table 1 was performed by combining the $40 \mathrm{mg}, 80 \mathrm{mg}$ and $120 \mathrm{mg}$ daily doses of febuxostat into a single category. The relative risk of cardiovascular adverse events (APTC and non-APTC combined) in febuxostat compared to allopurinol (200/300 mg) was 1.7 (95\% confidence interval (CI) $0.4,7.0)$ and compared to placebo was 2.5 (95\% CI $0.3,19.4$ ), with neither statistically significant (since the 95\% CI included one). The relative risks for mortality and serious adverse events was 1.0 (95\% CI $0.1,12.8)$ and 0.9 (95\% CI 0.5, 1.7), respectively, for febuxostat compared to allopurinol; for febuxostat compared to placebo, these were not estimable (no deaths occurred in either group) and 2.5 (95\% CI 0.7, 9.0), respectively. Although none of these comparisons were statistically significant, there was a non-significant trend towards more serious adverse events in febuxostat compared to placebo, but no difference when compared to allopurinol. A limitation of this meta-analysis is the presence of significant heterogeneity (as assessed by $\mathrm{I}^{2}$ statistic of $>50 \%$ ): cardiovascular adverse events $\left(\mathrm{I}^{2}, 48 \%\right)$, mortality $\left(\mathrm{I}^{2}, 58 \%\right)$ and serious adverse events $\left(\mathrm{I}^{2}, 41 \%\right)$. A 5-year open-label study [3] of patients taking febuxostat 40 to $120 \mathrm{mg}$ found $18 \%$ (21 of 116) suffered serious adverse events and 5\% (6 of 116) suffered cardiovascular adverse events. Another limitation is that outcomes were assessed at different time points for adverse events due to varying lengths of RCTs.

The data regarding safety presented in this study are important. Associations of hyperuricemia with cardiovascular risk are well known [11]. In addition, recent evidence also suggests that gout is an independent risk factor for overall cardiovascular mortality [12] and for myocardial infarction, after adjusting for hyperuricemiaassociated risk [13]. So, why would febuxostat, a medication that lowers serum urate (a cardiac risk factor), 
increase cardiac adverse events? There are perhaps two important issues that need to be addressed before we seek answers to this question: first, is the cardiovascular risk increased with febuxostat treatment compared to allopurinol or other urate-lowering agents, as was suspected with the initial RCT [5]? And second, is uratelowering below a certain level $(<4$ or $<3 \mathrm{mg} / \mathrm{dl}$ or some threshold) undesirable since lowering urate too much or too rapidly may create oxidative stress that could predispose to cardiovascular outcomes? We need welldesigned large database or registry post-marketing surveillance studies with validated, adjudicated cardiovascular outcomes to answer these important questions. While randomized studies of febuxostat with safety as the primary outcome would also be helpful, clinical trials often are of insufficient size, duration of follow-up, or have patients with insufficient generalizability to address such questions. It is possible that 'J' or ' $U$ ' shaped curves similar to ones observed for blood pressure and stroke risk [14] and diastolic blood pressure and cardiovascular outcomes in patients with coronary artery disease [15] may apply to serum urate and cardiovascular risk as well. In this exciting era of availability of new gout treatments and high-quality epidemiological and outcomes studies in gout, several existing questions will (need to) be answered and several new ones will likely emerge.

\section{Abbreviations}

APTC = Adjudicated Antiplatelet Trialists Collaboration; Cl = confidence

interval; $\mathrm{RCT}=$ randomized controlled trial.

\section{Competing interests}

There are no financial conflicts related to this work. No financial support was received for writing this editorial. JAS has received speaker honoraria from Abbott; research and travel grants from Allergan, Takeda, Savient, Wyeth and Amgen; and consultant fees from Savient, URL pharmaceuticals and Novartis.

\section{Acknowledgements}

This material is supported by the resources and the use of facilities at the Birmingham VA Medical Center, Alabama, USA. The views expressed in this article are those of the author and do not necessarily reflect the position or policy of the Department of Veterans Affairs or the United States government.

\section{Author details}

'Medicine Service, Birmingham VA Medical Center and Department of Medicine, University of Alabama, Birmingham, AL 35294, USA. ${ }^{2}$ Center for Surgical Medical acute Care Research and Transitions, Birmingham VA Medical Center, Birmingham, AL 35205, USA. ${ }^{3}$ Division of Epidemiology, School of Public Health, University of Alabama, Birmingham, AL 35294, USA. ${ }^{4}$ Departments of Health Sciences Research and Orthopedic Surgery, Mayo Clinic School of Medicine, Rochester, MN 55905, USA.

Published: 24 September 2010
References

1. Becker MA, Schumacher HR, Espinoza LR, Wells AF, Macdonald P, Lloyd E, Lademacher $C$ : The urate-lowering efficacy and safety of febuxostat in the treatment of the hyperuricemia of gout: the CONFIRMS trial. Arthritis Res Ther 2010, 12:R63.

2. Becker MA, Schumacher HR, MacDonald PA, Lloyd E, Lademacher C: Clinical efficacy and safety of successful longterm urate lowering with febuxostat or allopurinol in subjects with gout. J Rheumato/ 2009, 36:1273-1282.

3. Schumacher HR Jr, Becker MA, Lloyd E, MacDonald PA, Lademacher C: Febuxostat in the treatment of gout: 5-yr findings of the FOCUS efficacy and safety study. Rheumatology (Oxford) 2009, 48:188-194.

4. Shoji A, Yamanaka $H$, Kamatani N: A retrospective study of the relationship between serum urate level and recurrent attacks of gouty arthritis: evidence for reduction of recurrent gouty arthritis with antihyperuricemic therapy. Arthritis Rheum 2004, 51:321-325.

5. Becker MA, Schumacher HR Jr, Wortmann RL, MacDonald PA, Eustace D, Palo WA, Streit J, Joseph-Ridge N: Febuxostat compared with allopurinol in patients with hyperuricemia and gout. N Engl J Med 2005, 353:2450-2461.

6. Becker MA, Schumacher HR Jr, Wortmann RL, MacDonald PA, Palo WA, Eustace $D$, Vernillet $L$, Joseph-Ridge N: Febuxostat, a novel nonpurine selective inhibitor of xanthine oxidase: a twenty-eight-day, multicenter, phase II, randomized, double-blind, placebo-controlled, dose-response clinical trial examining safety and efficacy in patients with gout. Arthritis Rheum 2005, 52:916-923.

7. Schumacher HR Jr, Becker MA, Wortmann RL, Macdonald PA, Hunt B, Streit J, Lademacher C, Joseph-Ridge N: Effects of febuxostat versus allopurinol and placebo in reducing serum urate in subjects with hyperuricemia and gout: a 28-week, phase III, randomized, double-blind, parallel-group trial. Arthritis Rheum 2008, 59:1540-1548.

8. Perez-Ruiz F, Alonso-Ruiz A, Calabozo M, Herrero-Beites A, Garcia-Erauskin G, Ruiz-Lucea E: Efficacy of allopurinol and benzbromarone for the control of hyperuricaemia. A pathogenic approach to the treatment of primary chronic gout. Ann Rheum Dis 1998, 57:545-549.

9. Sarawate CA, Brewer KK, Yang W, Patel PA, Schumacher HR, Saag KG, Bakst AW: Gout medication treatment patterns and adherence to standards of care from a managed care perspective. Mayo Clin Proc 2006, 81:925-934.

10. Singh JA, Hodges JS, Asch SM: Opportunities for improving medication use and monitoring in gout. Ann Rheum Dis 2009, 68:1265-1270.

11. Johnson RJ, Kang DH, Feig D, Kivlighn S, Kanellis J, Watanabe S, Tuttle KR, Rodriguez-Iturbe B, Herrera-Acosta J, Mazzali M: Is there a pathogenetic role for uric acid in hypertension and cardiovascular and renal disease? Hypertension 2003, 41:1183-1190.

12. Krishnan E, Svendsen K, Neaton JD, Grandits G, Kuller LH: Long-term cardiovascular mortality among middle-aged men with gout. Arch Intern Med 2008, 168:1104-1110.

13. Krishnan E, Baker JF, Furst DE, Schumacher HR: Gout and the risk of acute myocardial infarction. Arthritis Rheum 2006, 54:2688-2696.

14. Voko Z, Bots ML, Hofman A, Koudstaal PJ, Witteman JC, Breteler MM: J-shaped relation between blood pressure and stroke in treated hypertensives. Hypertension 1999, 34:1181-1185.

15. Denardo SJ, Anderson RD, Pepine CJ: Blood pressure targets after high-risk myocardial infarction: is it time to update the guidelines? Hypertension 2008, 51:26-27.

doi:10.1186/ar3110

Cite this article as: Singh JA: Advances in gout: some answers, more questions. Arthritis Research \& Therapy 2010, 12:136 